\title{
Traducción entre lenguajes simbólicos de distintas áreas del conocimiento: el caso del flujo del campo eléctrico
}

\section{Translation between symbolic languages in different content areas: the case of the electric field flux}

\author{
M. Cecilia Pocoví, C. Collivadino \\ Universidad Nacional de Salta. Facultad de Ingeniería. Agencia de Promoción Científica y Tecnológica \\ Avda. Bolivia, 5150 (4400), Salta \\ cpocovi@unsa.edu.ar, collivad@unsa.edu.ar
}

\begin{abstract}
RESUMEN • Se presenta un estudio de caso genérico (Merriam, 1998) que consta de dos partes. En la primera se compara el sistema simbólico utilizado en la presentación del concepto de flujo de campo eléctrico en los textos de electromagnetismo de nivel universitario básico con aquel utilizado en los textos de cálculo matemático en la presentación del teorema de Gauss. El modelo de codificación dual (Sadoski y Paivio, 2004) provee el marco teórico para el análisis. Los resultados muestran que existe bastante uniformidad en el uso de símbolos en cálculo y en física pero existen discrepancias entre las dos áreas. En la segunda parte, se detecta el grado de traducción simbólica-simbólica que logra un grupo de estudiantes antes de realizar problemas de física sobre el tema. Se sugiere que la falta de una buena interpretación de los símbolos puede contribuir inicialmente a dificultar la comprensión de la idea de flujo de los estudiantes.
\end{abstract}

PALABRAS CLAVE: concepto de flujo; sistema simbólico; textos de Física; aprendizaje de Física; electrostática.

ABSTRACT • A generic case study as that defined by (Merriam, 1998) is presented here. In the first part of the work, the symbolic system used in basic electromagnetism books when presenting the concept of electric flux is compared with the symbolic system used in calculus books when presenting Gauss' Theorem. The analysis was guided by the Dual Coding Theory posed by Sadoski \& Paivio (2004). The results show that, while there exists a great deal of uniformity within one area (Physics or Calculus), there exist discrepancies between areas. The second part of this work deals with the symbolic-symbolic translation that students are able to carry out when having learned Gauss Theorem with a symbolic system that is different from the one used in Physics. It is suggested that the lack of interpretation of what the symbols mean can initially contribute to hinder comprehension about the flux concept.

KEYWORDS: flux concept; symbolic system; Physics texts; Physics learning; electrostatics.

Fecha de recepción: junio 2012 • Aceptado: noviembre 2013

Collivadino, C., Pocoví, M.C. (2014) Traducción entre lenguajes simbólicos de distintas áreas del conocimiento: el caso del flujo del campo eléctrico. Enseñanza de las Ciencias, 


\section{INTRODUCCIÓN}

La enseñanza y el aprendizaje de la electricidad y el magnetismo a nivel universitario básico ha sido objeto de estudio por parte de los investigadores en enseñanza de ciencias de todo el mundo. El foco inicial de dichas investigaciones lo constituyeron el aprendizaje y la enseñanza de los circuitos simples, foco que con el tiempo se fue ampliando a temas como la electrostática, el magnetismo y la electrodinámica de campos.

Los resultados de estos estudios representan un gran avance en el conocimiento específico de las ideas previas y persistentes que los estudiantes poseen sobre los distintos temas. También han aportado nuevas opciones didácticas elaboradas siguiendo diferentes fundamentos teóricos que han hecho posible un análisis todavía más detallado y profundo de los procesos cognitivos involucrados en el aprendizaje del electromagnetismo.

Los avances mencionados dan pie al planteamiento de nuevas preguntas acerca de los problemas de aprendizaje detectados. En este trabajo analizaremos el lenguaje simbólico utilizado en los libros texto de física de nivel universitario básico y su relación con el lenguaje matemático utilizado en cursos de cálculo previos a los de física. Específicamente, se abordará el concepto de flujo de campo eléctrico, que es el primer tema en el que se hace uso de conceptos matemáticos como integrales de superficie, flujo, diferenciales de área y normales a superficies, entre otros. El concepto de flujo es indispensable para lograr una comprensión cabal de la ley de Gauss en electrostática.

\section{ESTADO DEL ARTE}

Existen investigaciones que han abordado el estudio de los problemas que tienen los alumnos para comprender la ley de Gauss y los conceptos involucrados en ella, como, por ejemplo, el campo eléctrico y su flujo. Se mencionan, a continuación, algunos de los trabajos más relevantes sobre este tema y sobre otros conceptos estrechamente relacionados.

Un concepto básico involucrado en la idea de flujo del campo eléctrico es, justamente, el de campo eléctrico en sí. Se han realizado numerosas investigaciones que dan cuenta de distintos aspectos problemáticos en el aprendizaje del concepto de campo eléctrico. Furió y Guisasola (1998) mostraron que los alumnos prefieren utilizar un razonamiento basado en fuerzas de acción a distancia en lugar de analizar los problemas desde la teoría de campo. Viennot y Rainson $(1992,1999)$ estudiaron la comprensión de los alumnos acerca de la superposición de campos eléctricos. Sus resultados confirmaron las dificultades esperadas en el trabajo con el teorema de Gauss. Las mismas autoras, en 1999, presentaron el diseño y evaluación de una secuencia de enseñanza basada en las formas de razonamiento de los estudiantes detectados previamente. El análisis de las dificultades ontológicas y epistemológicas de los estudiantes en su aprendizaje del concepto de campo y sus formas de representación también ha sido realizado mediante estudios históricos acerca de la construcción del concepto (Furió y Guisasola, 1997; Pocoví y Finley, 2003, y Pocoví, 2007).

Guisasola et al. (2003) estudiaron distintos tipos de razonamiento de los estudiantes universitarios en situaciones referidas a la ley de Gauss (que involucra el flujo del campo eléctrico) y a la ley de Ampère (que involucra la circulación del campo magnético). Su trabajo caracteriza detalladamente algunas causas de las deficiencias en la comprensión y uso de estas leyes: además de las preconcepciones, señalan a las metodologías de razonamiento basadas en el "sentido común" como conducentes a situaciones de "reducción funcional" y "fijación funcional" (p. 197). También detectan problemas de comprensión de la ontología de las magnitudes físicas involucradas. Resaltan, además, la necesidad de que los docentes conozcan este tipo de dificultades de aprendizaje para poder así diseñar estrategias de enseñanza más efectivas. 
Krapas, Alves y Carvalho (2000) analizaron los modelos mentales que los estudiantes construyen sobre problemas referidos al flujo del campo eléctrico; en particular, el estudio sirvió para describir el dinamismo de estos modelos, su inestabilidad relativa y su forma representacional (proposicional y de imágenes). Guisasola, Almudi y Zuza (2011) logran un avance significativo al estudiar la comprensión de la inducción electromagnética de alumnos universitarios, concepto que involucra no solo la idea de flujo (magnético, en este caso), sino también su variación.

También existen trabajos que presentan propuestas didácticas para superar los problemas de aprendizaje como los detectados en las investigaciones anteriores. Por ejemplo, Alvárez (2009) muestra cómo se pueden utilizar recursos informáticos para visualizar el concepto de flujo involucrado en la ley de Gauss. Schneider (2004) hace uso de una analogía entre situaciones de flujo laminar y situaciones electrostáticas para enseñar a los estudiantes esta ley. Saarelainen y Hirvonen (2009) proponen una secuencia didáctica basada en la técnica de reconstrucción educacional que enfatiza la explicitación de la conexión entre la estructura del contenido que se quiere enseñar, los resultados de las investigaciones en enseñanza-aprendizaje y el desarrollo y la evaluación de la instrucción realizada.

Las investigaciones previas mencionadas se centran en la comprensión de conceptos físicos. Estos trabajos han realizado aportes fundamentales para esclarecer muchos problemas de aprendizaje del concepto de flujo y de la ley de Gauss. Sin embargo, sus abordajes poseen un matiz en común: se han desarrollado sin analizar ni cuestionar si la simbología matemática presentada en las clases de física es única o si es compartida o compatible con la simbología matemática aprendida previamente por los alumnos en clases de cálculo. En otras palabras, estos trabajos de investigación en enseñanza de física limitan su mención y análisis a la simbología y metodología de trabajo que es común a todos los textos de física. En este sentido, se puede decir que se está olvidando que los alumnos de nivel universitario han tenido su primer contacto con los símbolos involucrados en la definición de flujo de un campo vectorial en cursos previos de matemática.

El reconocimiento y la comprensión cabal de los símbolos utilizados para presentar un tema de física resultan de suma importancia para el correcto aprendizaje por parte de los alumnos. Existen trabajos en la literatura científica que tienen como foco los problemas de aprendizaje relacionados con la traducción entre distintos sistemas de codificación de la información científica. Por ejemplo, Bandiera et al. (1995) analizan las habilidades de "transposición de lenguajes" (verbal a algebraico, verbal a gráfico y algebraico a verbal) existentes en alumnos del último año de la escuela secundaria. Detectan que los logros alcanzados por dichos alumnos en relación con las habilidades de transposición son bajos respecto a lo que se espera de ellos al comenzar sus estudios universitarios. Sherin (2001) realiza un detallado estudio acerca de la comprensión de las ecuaciones de física en términos de "formas simbólicas" (p. 482) constituidas por la plantilla específica de símbolos (symbol template, p. 490) y los esquemas conceptuales (p. 491). Para ello, analiza la resolución de problemas por parte de estudiantes universitarios de segundo año. En sus conclusiones revaloriza la enseñanza más significativa de la física con ecuaciones (p. 523) y reniega de la "reputación negativa" (p. 524) de este tipo de abordaje entre los investigadores en enseñanza de ciencias.

Meredith y Marrongelle (2008) retoman el planteamiento realizado por Sherin y lo extienden al contexto de la resolución de problemas que requieren nociones de cálculo. Así, analizan los recursos utilizados por los estudiantes universitarios cuando deben realizar la operación de integración, en el caso específico de situaciones electrostáticas referidas a la densidad de carga y al campo eléctrico total en un punto del espacio. Detectan que muchos alumnos, aun cuando han estudiado el tema en clase y han sido evaluados en él, no entienden la integración como "la suma de efectos físicos infinitesimales para obtener un efecto físico total” (p. 576). Manogue et al. (2006) también enumera una serie de dificultades típicas que los alumnos poseen en el manejo de la integral curvilínea en problemas que involucran la ley de Ampere. 
Los trabajos mencionados en el párrafo anterior señalan serias dificultades de comprensión de los conceptos físicos que se manifiestan en la resolución de problemas que involucran la interpretación física de ecuaciones matemáticas. Las carencias detectadas son identificadas una vez que los alumnos han recibido la instrucción correspondiente e incluso ya han sido evaluados. Es decir, los resultados apuntan a mostrar deficiencias que persisten incluso después de la instrucción en la cual el alumno ha manipulado las ecuaciones. Cabría preguntar qué sucede cuando el alumno se encuentra por primera vez con una ecuación del tipo de la estudiada en cursos de matemática, en una clase de física.

El aprendizaje a partir de textos es una de las actividades más importantes en la educación en ciencias (Campanario y Moya, 1999; Concari y Giorgi, 2000; Pandiella, Torné y Macías 2004) y, en particular, en la educación en el nivel universitario (Kelly, 2007). En este trabajo estudiaremos los problemas de comprensión que pueden surgir en el primer contacto que el alumno tiene con un texto referido a la idea de flujo expresado en una ecuación. Dichos problemas pueden generarse a partir de una falta de uniformidad entre la simbología usada en los libros de física y aquellos de cálculo que han sido utilizados previamente por los estudiantes.

El Modelo de Codificación Dual (DCT, Dual Coding Theory) (Sadoski y Paivio, 2004) fue planteado inicialmente en estudios sobre la cognición en general y fue luego extendido a estudios sobre lectura. Este modelo supone que "todas las representaciones mentales retienen algunas cualidades concretas de las experiencias de las cuales derivan" (p. 1330). Estas experiencias pueden ser lingüísticas y no lingüísticas. Sus características distintivas se desarrollan en dos sistemas o códigos mentales: uno que procesa el lenguaje (código verbal) y otro que procesa los objetos no lingüísticos o eventos (código no verbal). En esta misma línea de investigación, Alexander y Kulicowich (1994) y Alexander y Jetton (2000) plantearon su modelo de aprendizaje a partir de textos y afirman en él que una característica distintiva de los textos de matemática y física es que consisten en modos de inscripción variados que corresponden a símbolos matemáticos e información lingüística. Esta coexistencia de modos de inscripción (o de sistemas) fue calificada por Bassok y Holyoak (1989) como bilingüe: mientras que el primer sistema consiste en fórmulas, gráficos y esquemas, el segundo está representado por frases y proposiciones que describen la situación física bajo estudio. Los requerimientos de procesamiento de información leída aumentan cuanto menos abundantes y explícitas son las traducciones de un sistema a otro en el texto (p. 900).

Según el modelo DCT, existen tres dimensiones o niveles de procesamiento de la información. La primera dimensión es llamada procesamiento representacional, y en ella el sujeto reconoce la familiaridad de algo, aunque ese reconocimiento no implique una comprensión cabal. Tanto en la segunda como en la tercera dimensión, el sujeto asocia una experiencia con alguna otra. En la segunda dimensión, el sujeto logra que la activación se extienda a otras representaciones en el mismo código, mientras que en la tercera extiende la activación a otras representaciones en códigos diferentes. Sadoski y Paivio (2004) presentan un ejemplo de los distintos niveles para el caso particular de aprendizaje a través de la lectura, aclarando que esta teoría es válida para situaciones más generales de cognición.

\section{PREGUNTAS DE ESTA INVESTIGACIÓN}

Los estudios mencionados implican que el aprendizaje del concepto de flujo de campo eléctrico en las clases de física presenta un nivel de dificultad alto: por un lado, se hace uso de un lenguaje simbólico correspondiente a herramientas matemáticas avanzadas para describir los fenómenos involucrados y, por otro lado, el sistema lingüístico que traduce los símbolos se refiere a conceptos abstractos como lo es el concepto de campo (Pocoví, 2007). 
En el presente trabajo nos preguntamos si, además de la complejidad propia recién mencionada y asociada con la traducción entre dos sistemas de diferente naturaleza (simbólico y lingüístico), el caso particular de la presentación del concepto de flujo del campo eléctrico tanto en los textos como en las clases posee una complejidad adicional que tiene que ver con la primera y segunda dimensión referidas en el DCT. En un primer contacto con la expresión simbólica del flujo de campo eléctrico, dichas dimensiones están asociadas a una traducción simbólica-simbólica. Es decir, el primer paso para interpretar una ecuación que describe un concepto es la identificación de los símbolos y el reconocimiento de lo que ellos representan basada en lo que el aprendiz conoce. Un estudio de esta situación implica, primero, analizar la simbología presentada en los textos de física y de cálculo y, segundo, detectar si, efectivamente, las diferencias encontradas en los símbolos pueden afectar a la comprensión inicial del concepto. Puesta en forma de preguntas de investigación, la complejidad recién descripta se expresa como:

1. ¿Existen diferencias en la simbología involucrada en la definición de flujo en los libros de texto de física y los de cálculo utilizados en las asignaturas universitarias bajo análisis?

2. ¿Logran los alumnos reconocer como familiares los símbolos utilizados en la definición de flujo campo eléctrico? En otras palabras, ¿logran identificar el símbolo integral utilizado en física como el equivalente al símbolo de integral sobre una superficie aprendida en cálculo? ¿Identifican cada uno de los factores dentro de la integral con la notación usada previamente en matemática?

3. Los símbolos representan conceptos y operaciones. Después de un primer contacto con los símbolos, es natural asociar a ese símbolo con algún tipo de operatoria que permite la representación completa del concepto. Entonces, es menester preguntar si los alumnos logran extender las nuevas representaciones (utilizadas en física) a las representaciones ya conocidas (aprendidas en los cursos de cálculo). O sea, una vez que nombran cada símbolo, ¿qué representa?, ¿qué trabajo operacional implican para lograr una representación completa de la idea de flujo?

Ambas preguntas están cercanamente ligadas a la traducción que los alumnos deben realizar entre los símbolos nuevos presentados en las clases de física y aquellos ya conocidos y trabajados en las clases de cálculo. Se debe notar que la importancia de estas dos dimensiones o niveles de procesamiento radica en que deben lograrse previamente a la comprensión de los conceptos físicos involucrados. En otras palabras, constituyen una condición necesaria (aunque no suficiente) para poder avanzar en el conocimiento cabal del tema, ya que en ellas el alumno reconoce la herramienta matemática que se utilizará para describir la física involucrada.

Generalmente, el concepto de flujo de un campo vectorial se enseńa inmediatamente antes de la ley de Gauss en electrostática, que es la primera ley de Maxwell que se presenta en los cursos de electromagnetismo de nivel universitario. Específicamente, en las carreras de ingeniería de las universidades argentinas se requiere que los alumnos aprueben un curso de cálculo en el que se presentan los teoremas integrales, como los de Gauss, Stokes y Green, antes de acceder a los cursos de electromagnetismo. El primero de los teoremas implica el cálculo de flujos de campos vectoriales. Dicho requerimiento tiene el propósito de posibilitar la comprensión de las leyes de Maxwell en forma integral y diferencial. Sin embargo, si estas herramientas y procedimientos matemáticos son aprendidos con simbología distinta a la utilizada en física, la comprensión de las leyes del electromagnetismo involucrará, además de la traducción simbólico-lingüística, una traducción entre dos sistemas simbólicos: el aprendido en los cursos de matemática previos y el utilizado en los cursos de física y presente en los textos de esta ciencia.

El presente trabajo consta de dos partes. En la primera, se compara el sistema simbólico utilizado en la presentación del concepto de flujo del campo eléctrico en forma integral en los textos de electromagnetismo de nivel universitario básico y el sistema simbólico utilizado en los textos de cálculo en la 
presentación del teorema de Gauss. Esta parte del trabajo permitirá la identificación de las diferencias de notación que pueden constituir escollos para lograr el reconocimiento y la extensión de los símbolos utilizados en una y otra área (primera pregunta de investigación). En la segunda parte, se analizan las respuestas de un grupo de alumnos universitarios a un ejercicio diseñado para detectar el grado de traducción simbólica-simbólica que logran en un primer encuentro con la expresión matemática del flujo tal como se expresa en muchos textos de física. En esta parte se comprobará si, efectivamente, las diferencias en simbología identificadas en la primera parte pueden afectar negativamente a la comprensión inicial del tema de física en los alumnos (segunda y tercera pregunta de investigación). En la realización de este ejercicio participan los alumnos de una cohorte de estudiantes de ingeniería de una universidad argentina.

\section{PRIMERA PARTE: ANÁLISIS DE LOS LIBROS DE TEXTO}

\section{Metodología utilizada en la selección y análisis de los textos}

Ya que el problema planteado ha sido descrito en la sección anterior, a continuación se explicará cómo se seleccionó la unidad de análisis de este estudio de caso, es decir, qué constituye la muestra de libros seleccionados para realizar este estudio. Para ello se utilizó la estrategia no probabilista más apropiada (según Merriam, 1998). llamada "muestra seleccionada con un propósito" (purposeful sampling) según Patton (1990). Más específicamente, corresponde a una "muestra típica”, es decir, a una que refleja una situación promedio o común en el fenómeno estudiado (Merriam, 1998: 62).

Como el objetivo primordial en esta parte del trabajo es comparar el sistema simbólico utilizado en las clases de cálculo de las carreras de ingeniería de una universidad argentina con aquel utilizado en los cursos de física de electromagnetismo básico, el conjunto de libros analizados corresponde a aquellos recomendados por los profesores a cargo de las asignaturas correspondientes. Dichos libros figuran también como recomendados en los programas académicos de las mismas asignaturas y son los pedidos con mayor frecuencia en los servicios de biblioteca de la universidad.

Somos conscientes de las limitaciones respecto a la generalización propias de un estudio de caso tal como el presente, pero tenemos el convencimiento de que el análisis realizado y sus conclusiones servirán a los lectores (otros investigadores y profesores) para, posiblemente, encontrar hasta dónde estos hallazgos se aplican a otras situaciones similares. A este tipo de situación, Merriam (1998) la denomina "generalización del lector o del usuario" (p. 211).

El protocolo para el análisis de los libros se llevó a cabo también teniendo en cuenta el objetivo de esta parte del estudio. Es decir, se identificaron los distintos símbolos que se involucran en la presentación del concepto de flujo de un campo vectorial tanto en el área de matemática como en la de física y se realizó la comparación entre ellos.

El análisis fue realizado por dos investigadores independientes y los datos fueron cruzados en reuniones comunes. Las pocas discrepancias encontradas fueron consensuadas en dichas reuniones, ya que consistían en la omisión de algún aspecto de las definiciones presentadas en los libros por parte de alguno de los investigadores.

\section{Análisis de la simbología utilizada en los textos de física}

El concepto de flujo de campo eléctrico es generalmente presentado en los libros inmediatamente antes que la ley de Gauss, la cual, en su forma integral, establece que en el vacío el flujo neto del campo eléctrico a través de una superficie cerrada es igual a la carga encerrada en dicha superficie dividida la permitividad eléctrica del vacío. 
Los símbolos involucrados tanto en la expresión matemática de esta ley como en la definición de flujo corresponden a una integral de superficie, al vector campo eléctrico, a un producto escalar y a un diferencial de área. Este último puede escribirse como un diferencial de área que apunta en la dirección normal a la superficie de integración y hacia afuera de esta o como un diferencial de área (en módulo) multiplicado por el versor normal a la superficie.

Para analizar los libros de física de nivel universitario básico elaboramos un primer registro en el cual se detallaron los símbolos utilizados en la presentación del concepto de flujo y de la ley de Gauss. En un segundo registro identificamos los nombres con que se describen los símbolos del primer registro y, en el caso particular del símbolo del producto escalar, también anotamos cómo se lleva a cabo la operatoria correspondiente a él. Si bien parte de este segundo registro involucra un aspecto lingüístico (nombre de símbolos), este se halla limitado a aquellas expresiones con las que se denomina cada símbolo en el texto.

En lo que sigue se presentan los resultados, y en ellos se han asignado (por cuestiones de espacio) los siguientes números a los libros citados de manera completa en las referencias de este trabajo: [1] Young y Freedman (2009), [2] Resnick, Halliday y Krane (2009), [3] Purcell (1973), [4] Serway y Jewett (2009), [5] Tipler y Mosca (2010), [6] Alonso y Finn (1976), [7] Wangsness (1986), [8] Halliday y Resnick y [9] Kip (1975).

Primer registro. Símbolos utilizados:

a) Símbolo para la integral: los libros [1], [2], [4] y [8] utilizan el símbolo $\oint \square$ para referirse a la integral de superficie. El libro [9] introduce dos notaciones $\left(\iint_{s c}^{\square} \square, \int_{s c}^{\square} \square\right)$ y aclara que en ambos casos los símbolos se refieren a una integración sobre la superficie. El texto [3] utiliza el símbolo $\int$ ․ El resto utiliza el símbolo $\oint_{S}^{\square}$

b) Símbolo para el diferencial de área: los libros [1], [2], [3], [4] y [7] utilizan el símbolo $\overrightarrow{d A}$ o $\overrightarrow{d a}$, mientras que los libros [8] y [9] utilizan $\overrightarrow{d S}$. El [5] y [6] usan $d A$ y $d S$, respectivamente.

c) Símbolo para el versor normal: el símbolo $\hat{n}$ es utilizado por el libro [5] y el símbolo $\vec{u}_{N}$ por el libro [6]. El libro [1] presenta el versor $\hat{n}$ cuando define el concepto de flujo en una sección anterior, pero en la ley de Gauss trabaja directamente con el vector diferencial de área. El resto de los libros no desdobla el vector diferencial de área en el versor normal y la magnitud del diferencial de área considerado. De esta manera, no necesitan introducir explícitamente el versor normal en la integral. Se hace notar que ninguno de los libros explicita el versor normal en componentes según los ejes coordenados utilizados.

d) Símbolo para el producto: todos los libros utilizan el punto (.) como símbolo del producto escalar. Cuando explicitan lo que este símbolo representa en los ejemplos, todos optan por la multiplicación de los módulos de los vectores involucrados y el coseno del ángulo formado por ellos. Ninguno realiza la operación de manera analítica, multiplicando componente a componente los vectores involucrados.

e) Representaciones gráficas utilizadas: los libros que no introdujeron el desdoblamiento del vector diferencial de área muestran directamente este vector en las gráficas. Los libros [1], [5] y [6] representan los versores unitarios en los esquemas. Todos muestran a los vectores campo eléctrico en distintos puntos de la superficie.

f) Símbolo para el campo vectorial dentro de la integral: $\vec{E}$ o $E$. 
Segundo registro. Expresiones lingüísticas asociadas cada símbolo:

a) Todos los textos se refieren a" al nombrar el símbolo utilizado para la integración. Un solo libro [9] se refiere, en un pie de página, a la simplificación de la notación que se adopta al elegir escribir la integral de superficie con un solo símbolo integral.

b) El diferencial que aparece en las integrales se nombra como diferencial de área o de superficie en todos los libros. En la mayoría, este diferencial tiene carácter vectorial y no se desglosa en el producto del versor normal por un diferencial de área escalar. Los libros [5] y [6], en cambio, sí realizan dicha separación.

c) Los libros [1], [5] y [6] nombran al símbolo $\hat{n}$ o $\vec{u}_{N}$ como el versor perpendicular al diferencial de superficie, aunque, como se dijo en el primer registro, el libro [1] no utiliza dicho versor durante el trabajo con la ley de Gauss. Los libros restantes (incluido el [1] en el tratamiento de la ley de Gauss) no trabajan con el versor normal y asignan su dirección y sentido al vector diferencial de superficie.

d) Todos los libros nombran al producto punto como escalar.

e) Las explicaciones lingüísticas de los esquemas realizados se centran en todos los casos en la descripción del ángulo que forman los vectores campo eléctrico y el diferencial de área (o la normal si se usa en forma explícita).

f) Como ya dijimos, en física, tanto el concepto de flujo presentado en los libros como la ley de Gauss se refieren al cálculo del flujo del campo eléctrico a través de una superficie cerrada. Debemos destacar que el nombre campo eléctrico se usa en dos sentidos distintos que tal vez puedan aclararse si se hace referencia a otro caso de magnitud vectorial: la velocidad. Con la palabra velocidad nos referimos a un vector que se asocia a una partícula en un cierto punto del espacio. Cuando hablamos de campo de velocidades, en cambio, nos referimos a una función vectorial que describe en forma global alguna distribución de velocidades existente en las partículas en cierta región (por ejemplo, en un caño). En cambio, se dice campo eléctrico tanto para referirnos a un vector que es una propiedad de un punto del espacio, como para referirnos a la función vectorial que describe la situación eléctrica de una región del espacio. En otras palabras, como la magnitud tiene un nombre que ya incluye la palabra campo, sería bastante complicado hablar del campo del campo eléctrico. Es entendible, pues, que los libros de física se refieran directamente al flujo del campo eléctrico.

Como se desprende de los registros anteriores, se puede decir que existe una uniformidad bastante marcada en el tratamiento del concepto de flujo de campo eléctrico y de la ley de Gauss en los libros de física utilizados en cursos de electromagnetismo básico a nivel universitario. Si bien todos los libros utilizan un solo símbolo de integral para representar una integral de superficie, llama la atención que, excepto en un caso [9], no se menciona ninguna otra forma común de escribir la integral. Tampoco se menciona, ni en el texto ni en los ejemplos, el producto escalar como realizado componente a componente. Por último, todos los libros usan la frase campo eléctrico para referirse tanto al vector individual como a la función que muestra la situación eléctrica en cierta región del espacio.

\section{Análisis de la simbología utilizada en los textos de cálculo}

El teorema de Gauss presentado en los cursos universitarios de cálculo establece que el flujo de un campo vectorial a través de una superficie que limita un volumen es igual a la integral de la divergencia del campo en dicho volumen. 
Como nos interesa analizar los problemas iniciales de aprendizaje relacionados con la expresión integral del flujo del campo eléctrico en física, limitamos nuestro análisis a los símbolos involucrados en la parte del teorema que define el flujo de un campo vectorial. La metodología utilizada coincide con la descrita en el caso de los textos de física, es decir, se recabaron dos registros: uno correspondiente a los símbolos utilizados en la presentación del flujo y otro que corresponde a las expresiones lingüísticas que acompañan a cada símbolo utilizado.

En lo que sigue se presentan los resultados, y en ellos se han asignado los siguientes números romanos a los libros citados de manera completa en las referencias de este trabajo: [I] Leithold (1998), [II] Purcell, Varberg y Rigdon (2001), [III] Piskunov (1998), [IV] Stewart (2006), [V] Spinadel(2004), [VI] Hsu (1987), [VII] Spiegel (1970), [VIII] Rey Pastor (1968) y [IX] Rabuffetti (1984) y Apostol (1967).

Primer registro. Símbolos utilizados:

a) Símbolo para la integral: todos los libros utilizan el símbolo $\iint_{S}^{\square} \square$ para referirse a la integral de superficie, excepto el libro [VI], que indica el hecho de que la superficie es cerrada colocando un círculo sobrepuesto con las integrales $\left(\oiint_{S}^{\square}\right)$.

b) Símbolo para el diferencial de área: la mayoría de los libros usan los símbolos $d S, d \Sigma$ o $d \sigma$ para representar el diferencial de superficie. Dos de ellos ([IV] y [VI]) utilizan el símbolo del diferencial vectorial, aclarando que representa el versor normal a la superficie multiplicado por la magnitud del diferencial de superficie.

c) Símbolo para el versor normal: en todos los casos se utiliza un versor representado por una $n$ minúscula resaltado en negrita o con una flecha encima de la letra. Las componentes de este versor normal se escriben en función de los cosenos directores de los ángulos $\alpha, \beta$ y $\gamma$, que son los ángulos que forma la normal con los ejes $x, y$ y $z$, respectivamente.

d) Símbolo para el producto y trabajo analítico asociado: todos los libros utilizan el punto (.) como símbolo del producto escalar. Cuando explicitan este producto en símbolos, todos optan la explicitación de la operación de manera analítica, multiplicando las componentes de uno por las componentes correspondientes del otro.

e) Representaciones gráficas utilizadas: los libros [I], [III] y [VII] no muestran esquemas en la presentación del teorema. Algunos (IV y V) reservan los gráficos para la presentación de los ejemplos. Los esquemas realizados en algunos libros ( $\mathrm{IX}$ y X) son simples y se limitan a mostrar al lector el volumen encerrado por distintas superficies.

f) El campo del cual se calcula el flujo se simboliza con una letra (f, F, A, v) resaltada en negrita o con el símbolo de vector (pequeña flecha) arriba de la letra.

Segundo registro. Expresiones lingüísticas asociadas cada símbolo:

a) Todos los textos se refieren a la integral de superficie al nombrar el símbolo utilizado para la integración. Los libros ([I], [II], [III], [VII], [IX] y [X]) diferencian entre "integral de superficie" e "integral doble" explicando que la integral sobre una superficie alabeada se transforma a una integral doble para resolver. Algunos explicitan esta diferencia en el teorema de Gauss y otros, algunas secciones antes.

b) El diferencial que aparece en las integrales se nombra diferencial de superficie en todos los libros y generalmente ha sido presentado previo al tratamiento del concepto de flujo y el teorema de Gauss.

c) Todos los libros identifican con el nombre de versor normal al diferencial de superficie a los símbolos $n$ о $\vec{N}$ 
d) Todos los libros nombran al producto punto como escalar. Todos los libros resuelven la integral planteada mediante el producto escalar realizado componente a componente.

e) Las explicaciones lingüísticas de los esquemas realizados son escuetas y se refieren solo a la superficie que limita un volumen.

f) Todos los libros describen la integral como el cálculo del flujo de un campo vectorial a través de la superficie. Se define campo vectorial como una función vectorial.

Como se puede apreciar de los registros anteriores, se puede decir que también existe una uniformidad bastante marcada en el tratamiento del concepto de flujo en los libros de cálculo utilizados en cursos de nivel universitario. Todos los libros utilizan dos símbolos de integral para representar la integral de superficie y, además, varios hacen notar al lector que para la resolución la integral doble sobre una superficie alabeada debe transformarse en una integral de superficie. Cada vector involucrado en el producto escalar (la función vectorial o la normal a la superficie) se describe en forma y componentes. El producto escalar se escribe con el símbolo punto (.) y se realiza en los ejemplos mediante el producto componente a componente. Por último, la mayoría de los libros de cálculo definen primero una función vectorial o campo vectorial y establecen que van a calcular el flujo de dicho campo vectorial.

\section{Comparación entre la simbología y la descripción de los símbolos en los textos de física y los de cálculo}

A pesar de que se detectaron patrones uniformes en la simbología y en las descripciones lingüísticas de los símbolos, en los libros utilizados en la asignatura de electromagnetismo básico y aquellos utilizados en el curso de cálculo dichos patrones no coinciden.

Los símbolos utilizados para representar una integral de superficie son diferentes. Mientras que todos los textos de física usan un solo símbolo integral $\left(\int \square\right)$, los de cálculo utilizan dos $\left(\iint \square\right)$. Más aún, la mayoría de los libros de cálculo realizan la distinción entre una integral de superficie y una doble en la cual se deben realizar proyecciones en los planos coordenados.

El diferencial de superficie aparece en la mayoría de los libros de física analizados como un diferencial vectorial que tiene la dirección de la normal a la superficie y es de magnitud igual al tamaño del diferencial de superficie considerado. En cambio, en la mayoría de los libros de cálculo revisados se desdobla explícitamente la magnitud del diferencial de superficie y el versor normal.

En ningún caso los textos de física explicitan las componentes del vector diferencial de área o del versor normal en su trabajo con el concepto de flujo. Los textos de cálculo descomponen el versor normal en componentes que explicitan los ángulos con los ejes coordenados.

El símbolo usado para representar el producto escalar es el mismo tanto en física como en cálculo, pero mientras que en física el desarrollo de este producto se muestra usando los módulos de los vectores involucrados por el coseno del ángulo que forman, en cálculo dicho producto se realiza componente a componente.

Los libros de cálculo se refieren a la función vectorial en la integral de superficie del teorema de Gauss como un campo o función vectorial del cual se calcula el flujo. En los libros de física, el símbolo correspondiente al "campo eléctrico" se usa tanto para representar el vector en un punto como para referirse al campo o función vectorial del cual se calcula el flujo.

Como se puede apreciar, existen varias diferencias en la simbología y en los nombres asignados que podrían afectar al reconocimiento de los símbolos utilizados en la definición de flujo como familiares y también a la extensión de las nuevas representaciones (usadas en física) a las representaciones ya conocidas (estudiadas en los cursos de cálculo). En la segunda parte de este trabajo se determinará si las diferencias en simbología detectadas afectan negativamente a los dos primeros niveles del procesa- 
miento de la información, según la DCT, para el caso del aprendizaje del concepto de flujo de campo eléctrico en física.

\section{SEGUNDA PARTE: RECONOCIMIENTO DE LA EQUIVALENCIA ENTRE LOS TEXTOS DE FÍSICA Y LOS DE CÁLCULO}

La unidad de análisis en esta parte del trabajo está constituida por una cohorte de alumnos del curso de electromagnetismo correspondiente a las carreras de Ingeniería de una universidad argentina (69 alumnos). Todos los alumnos han aprobado el curso correlativo de cálculo. En este sentido, los alumnos constituyen una muestra típica o situación promedio del fenómeno estudiado (Merriam, 1998: 62), por ejemplo, del nivel de reconocimiento de los símbolos utilizados en física que logran dichos alumnos.

Alfassi (2004) determinó que, a medida que los estudiantes avanzan en sus estudios, los textos pasan a ser la fuente primordial de conocimiento. Ya que nuestro interés es identificar los problemas de comprensión que surgen cuando los estudiantes se encuentran por primera vez con el concepto de flujo del campo eléctrico, elegimos presentarles una serie de preguntas referidas a un texto que contiene exclusivamente la definición de "flujo del campo eléctrico a través de una superficie cerrada" y su expresión en símbolos. Si bien el flujo de un campo vectorial puede definirse también para una superficie abierta, elegimos centrarnos en el caso de una superficie cerrada por dos razones. Primero, este será el tipo de flujo que cobra importancia en el tratamiento posterior de la ley de Gauss y, por lo tanto, nos interesa ver el reconocimiento de los símbolos asociados al caso de una superficie cerrada tratado en dicha ley. Segundo, el cálculo del flujo a través de una superficie abierta presenta una indeterminación, ya que el sentido del vector normal a la superficie está indeterminado. La recolección de datos se realizó mediante el análisis de las respuestas dadas en forma escrita e individual.

Las preguntas planteadas a los alumnos son muy simples en cuanto a su contenido, ya que están apuntadas a detectar si los alumnos cumplen con las dos primeras dimensiones referidas en el DCT como paso previo a la comprensión de la física involucrada en la ley de Gauss. Es decir, las preguntas se plantearon para ver si los alumnos pueden reconocer los símbolos utilizados en la definición de flujo y si pueden extender las nuevas representaciones (utilizadas en física) a las representaciones ya conocidas (aprendidas en los cursos de cálculo). El enunciado se transcribe a continuación:

Compare la definición de flujo a través de una superficie cerrada que usted estudió en Análisis Matemático II $\left(\phi=\oiint_{\Sigma}^{\square} \vec{a} \cdot \vec{n} d \Sigma\right)$ con la definición que usaremos en Física II de la misma magnitud: $\phi=\oint_{S}^{\square} \vec{E} \cdot d \vec{S}$.

I. ¿A qué corresponde cada símbolo? Describa en palabras si existe una correspondencia entre los símbolos de una y otra expresión para el flujo. ¿Qué se entiende por cada uno? Tenga en cuenta tanto los símbolos que se refieren a operaciones como los que indican magnitudes.

II. Describa cómo realiza el producto simbolizado por un punto (.) entre dos vectores $\vec{A}$ y $\vec{B}$ ”.

Para el análisis de las respuestas al inciso I) se utilizó un protocolo centrado tanto en el reconocimiento de los símbolos (de integral, campo vectorial, normal a la superficie y diferencial de superficie), como en el nombre que se asigna a estos. Se listan a continuación los resultados de esta etapa.

a) Una primera clasificación de las respuestas se realizó teniendo en cuenta si los alumnos mencionaron o no el símbolo integral en sus respuestas. El primer grupo estuvo compuesto por el $16 \%$ de los alumnos $(\mathrm{n}=11)$, quienes no se refirieron en sus respuestas al símbolo integral. Los alumnos restantes, el $84 \%$ del total $(n=58)$, conforman un segundo grupo, que expresa que 
los símbolos integrales en las dos definiciones representan efectivamente una integral. El total de este segundo grupo de alumnos reconoce la integral de la definición trabajada en el curso de cálculo como una integral de superficie. Sin embargo, solo diez alumnos expresan que la integral en la definición del flujo presentada en física corresponde también a una integral de superficie. Algunos ejemplos de este tipo de respuestas son (en corchetes se escriben aclaraciones realizadas por las investigadoras): "creo que la integral $\oint_{S}^{\square} \vec{E} \cdot d \vec{S}$ debe ser de superficie pero la forma es otra" (alumno \#6), "la segunda [la integral usada en física] debe ser una integral de superficie porque la otra [la usada en matemática] es de superficie" (alumno \#29), "por analogía veo que tiene que ser de superficie porque así era en mate [matemática]" (alumno \#18). Sin embargo, treinta y un alumnos se refieren a la integral presentada en física como a una integral curvilínea expresando, por ejemplo: "La $\oint_{S}^{\square}$ a es una integral curvilínea alrededor de $S$ " (alumno \# 32); "En física se hace sobre la curva $S$ " (alumno \# 16). El resto (diecisiete alumnos) mencionan en su explicación que la expresión del flujo como definido en física involucra un solo símbolo integral o una integral simple, distinguiéndola, así también, de la integral de superficie en la definición de flujo presentada en cálculo. Por ejemplo, "la de física es una integral distinta de la que vimos antes [matemática] porque tiene un solo símbolo" (alumno \# 21).

b) En cuanto al campo vectorial, el 92\% de los alumnos identificó al $\vec{a}$ presentado en la expresión de cálculo como un campo vectorial, mientras que el resto no lo mencionó en su respuesta. El $76 \%$ de los alumnos del primer grupo se refirieron al campo eléctrico $\vec{E}$ como el equivalente al campo $\vec{a}$ de la primera expresión. Por ejemplo: "como las integrales tienen la misma pinta [forma] el $\vec{E}$ debe ser el $\vec{a}(x, y, z)$ de antes [de matemática]" (alumno \# 18). El 24\% restante no menciona esta equivalencia en sus repuestas. Resulta interesante notar que muchos estudiantes colocaron en sus explicaciones la dependencia con las coordenadas $x, y$ y $z$ al referirse al campo $\vec{a}$ (es decir, $\vec{a}(x, y, z))$ y, sin embargo, ninguno explicitó el campo $\vec{E}$ como una función de la posición.

c) Si bien el $100 \%$ de los alumnos empleó la palabra normal al referirse al $\vec{n}$ (que aparece en cálculo) y muchos la expresaron como $\vec{n}=\frac{\nabla F}{\| \nabla F \mid}$, solo diez alumnos reconocieron explícitamente la relación entre este vector y la dirección del diferencial de área vectorial que aparece en la expresión del flujo como presentado en física. Por ejemplo, "el $\vec{n}$ debe ser la normal del diferencial de superficie de física" (alumno \# 27), o "si comparo las dos, debe ser la normal a la superficie porque el $\overrightarrow{d S}$ es vector y el $d \Sigma$ no" (alumno \# 29).

d) El $83 \%$ de los alumnos reconoce en $d \Sigma$ un diferencial de superficie, pero de este grupo solo aquellos que también identifican la $\oint_{S}^{\square} \square$ como una integral de superficie explicitan que los dos diferenciales $(d \Sigma$ y $\overrightarrow{d S})$ representan diferenciales de superficie.

e) Todos los alumnos reconocen el símbolo "." con el nombre de producto escalar y al referirse a la operación.

En cuanto al inciso II), el 93\% de los alumnos describen a cada vector en componentes según los ejes coordenados $\left(\mathrm{A}_{\mathrm{x}}, \mathrm{A}_{\mathrm{y}}, \mathrm{A}_{\mathrm{z}}\right)$ y $\left(\mathrm{B}_{\mathrm{x}}, \mathrm{B}_{\mathrm{y}}, \mathrm{B}_{\mathrm{z}}\right)$, y explicitan este producto llevando a cabo la multiplicación componente a componente. Es decir, " $\left(A_{x}, A_{y}, A_{z}\right) .\left(B_{x}, B_{y}, B_{z}\right)=A_{x} B_{x}+A_{y} B_{y}+A_{z} B_{z}$ ” (alumno \# 17). Los restantes no dieron respuestas coherentes, por ejemplo, “ $\vec{A}$ por $\vec{B}$ " (alumno \#21) o " $\vec{A} \times \vec{B}$ " (alumno \# 1). Ningún alumno menciona la expresión que involucra el producto de los módulos por el coseno del ángulo formado por los vectores, que es la forma usada generalmente en los planteamientos de situaciones electrostáticas en la ley de Gauss. 
Como se puede observar, el nivel de reconocimiento de la simbología es rudimentario, ya que un gran porcentaje de alumnos no reconoce en el símbolo $\oint_{S}^{\square} \square$ la expresión de una integral sobre una superficie, a pesar de tener a la vista y haber trabajado previamente con el flujo en cursos de cálculo. Más aún, muy pocos explicitan la relación entre el vector normal y la dirección del vector diferencial de superficie en física.

Vale la pena recalcar nuevamente lo indicado en el inciso b): varios alumnos señalan la dependencia del campo vectorial "de cálculo" como dependiente de la posición, y no lo hacen para el caso del campo vectorial "de física". Si bien este aspecto, por sí solo, no alcanza para afirmar que muchos alumnos no interpretan inicialmente al campo como una función de la posición, este resultado nos pone en alerta sobre la existencia potencial de un problema que debería prevenirse en instancias posteriores de aprendizaje.

La operatoria relacionada con el símbolo de producto escalar también difiere respecto a cómo se plantea generalmente en los problemas posteriores de ley de Gauss, en los que se hace uso de la simetría de las situaciones analizadas para trabajar directamente con el producto de las magnitudes del campo y la superficie y el coseno de ángulo que forman.

\section{CONCLUSIONES}

Este trabajo muestra que, en un primer contacto con la simbología utilizada en clases de física para definir flujo del campo eléctrico, muchos alumnos no logran reconocer los símbolos utilizados en los textos de física como símbolos familiares (primera dimensión en el DCT), ni tampoco logran la extensión de las nuevas representaciones (utilizadas en física) a las representaciones ya conocidas (aprendidas en los cursos de cálculo). Si bien existen otras instancias posteriores de aprendizaje, estos resultados nos ponen en alerta sobre algunas cuestiones que deberían aclararse y resaltarse antes de seguir adelante con el estudio de la ley de Gauss.

Las palabras que describen la primera y segunda dimensión del DCT son reconocimiento y extensión, respectivamente. Estas palabras se usan en el contexto del re-encuentro e identificación del lector con alguna experiencia conocida previamente. En el caso de la integral de superficie, la mayoría de los alumnos demuestran haber retenido las características lingüísticas (el nombre) y las no lingüísticas (el símbolo) de dicha integral, como se estudió en los cursos de cálculo. Así pues, la mayoría reconoce la expresión del flujo visto en las clases de cálculo sin mayores problemas. Sin embargo, la presentación de la misma idea (flujo) mediante el uso de otra simbología, y aun cuando en un mismo texto se presenta la noción de flujo tal como se estudió previamente, resulta en situaciones de reconocimiento y extensión fallidas. Nótese que usamos el término fallidas en vez del de nulas, ya que la mayoría de los alumnos reconocen en el símbolo utilizado en física $\left(\oint_{S}^{\square} \square\right)$ como acorde con el símbolo de integral curvilínea usado en cálculo: un solo símbolo integral con una letra como subíndice. En otras palabras, el reconocimiento correcto de la expresión de flujo es impedido por dos factores: primero, se usa una simbología distinta a la aprendida anteriormente y, segundo, la simbología utilizada coincide con la utilizada previamente para otro concepto (integral sobre una curva). Hasta el punto de que en el segundo nivel del procesamiento de la información muchos estudiantes logran que la activación se extienda a otras representaciones $\left(\oint_{S}^{\square} \square\right)$ ) en el mismo código $\left(\oint_{C}^{\square} \square\right)$, y llegan a la conclusión de que la integral en la definición de flujo en física es una integral curvilínea.

Sin pretensiones de generalizar estos resultados, se puede afirmar que, en la muestra analizada, se hace patente el efecto multiplicativo que conlleva una primera identificación y extensión fallida del símbolo integral: los alumnos en esta situación no pueden reconocer el $\overrightarrow{d S}$ utilizado en física como 
el diferencial de superficie, y tampoco pueden relacionar el vector normal con el vector asociado a la superficie en el caso de física.

Otra situación detectada que podría generar problemas en un encuentro inicial con la expresión del flujo es la operatoria con el producto escalar entre dos vectores. Mientras que en las clases de cálculo se utiliza generalmente el producto componente a componente (como fue realizado por la mayoría de los alumnos), el planteamiento de las situaciones electrostáticas en la ley de Gauss es realizado mediante el producto de los módulos de los vectores intervinientes por el ángulo entre ellos. De nuevo, los alumnos podrían no reconocer la operación planteada en física si no se tiene cuidado en señalar la equivalencia con la forma conocida de cálculo.

En cuanto a la expresión del campo involucrado en la definición de flujo, el cambio de la letra que lo representa ( $\vec{E}$ por $\vec{a})$ no ha resultado en una identificación y extensión problemática. Sin embargo, llama la atención que, como se mencionó previamente, varios alumnos explicitaron la dependencia espacial del campo $\vec{a}$ como $\vec{a}(x, y, z)$, mientras que ninguno lo hizo al referirse al campo eléctrico. Sería interesante indagar en el futuro si efectivamente el alumno reconoce al campo eléctrico en la integral como función espacial. Este reconocimiento es de suma importancia, ya que, justamente, en los casos sencillos tratados en la ley de Gauss se hace uso de las propiedades de simetría espacial de $\vec{E}$ para poder trabajar de forma sencilla con las integrales de superficie.

Los profesores, a medida que nos acostumbramos a presentar distintos temas, a veces olvidamos que los alumnos están aprendiendo esos conceptos por primera vez. Si, en su contacto inicial con una idea, los alumnos no logran relacionarla con conceptos o temas conocidos, su aprendizaje se tornará innecesariamente problemático. No podemos perder de vista que nuestro objetivo final es lograr que nuestros alumnos aprendan la física por detrás de la expresión matemática de un concepto, pero ¿̨cuánto daño hacemos si permitimos que la falta de reconocimiento inicial de símbolos afecte al aprendizaje?

Es de esperar que investigaciones futuras ayuden a contestar a esta pregunta realizando estudios en otros temas y planteando abordajes que lo remedien. Cualquier solución a este tipo de situaciones involucrará la coordinación entre los profesores de cálculo y de física apuntada a ayudar a los alumnos a lograr el procesamiento inicial de la información presentada. Una posibilidad que estamos planteando en este momento es la del diseño de un texto en el cual el planteamiento analítico de las primeras situaciones estudiadas en la ley de Gauss en electrostática (carga puntual y carga esférica) se describen usando un paralelismo entre la manera de tratar el problema como fue aprendido en las clases de cálculo y la manera en que se usará en las clases de física. Esta no es una tarea fácil ni obvia pero sí es muy enriquecedora, ya que en la misma interacción entre docentes de distintas áreas se pueden descubrir algunos problemas de comunicación que surgen, justamente, de la diferencia en la forma de representar un mismo concepto.

\section{REFERENCIAS}

Alexander, P. A. y Kulicowich, J. M. (1994). Learning from a Physics text: A Synthesis of recent research. Journal of Research in Science Teaching, 31(9), pp. 895-911. http://dx.doi.org/10.1002/tea.3660310906

Alexander, P. A. y Jetton, T. L. (2000). Learning from Text: A multidimensional and developmental perspective. En Kamil, Mosenthal, Pearson y Barr (eds.). Handbook of Research of Reading Research, vol III, pp. 285-311. NJ: LEA, Inc.

Alfassi, M. (2004). Reading to learn: Effects of combined strategy instruction on high school students. The Journal of Educational Research, 97(4), pp. 171-184.

http://dx.doi.org/10.3200/JOER.97.4.171-185 
Alonso, M. y Finn, E. J. (1976). Física. Campos y Ondas. Vol. 2. México: Fondo Educativo Interamericano, S.A.

Álvarez, T. (2009). Uso de estrategias didácticas basadas en las TIC para la visualización de conceptos involucrados en el aprendizaje del electromagnetismo, en estudiantes de ingeniería. Enseñanza de las Ciencias, número extra, VIII Congreso Internacional sobre Investigación en Didáctica de las Ciencias, Barcelona, pp. 552-558.

Apostol, T. M. (1967). Calculus. Volumen II. Buenos Aires: Editorial Reverté, S. A.

Bandiera, M.; Duprè, F.; Ianniello, M. G. y Vicentini, M. (1995). Una investigación sobre habilidades para el aprendizaje científico. Enseñanza de las Ciencias, 13(1), pp. 46-54.

BAssok, M. y HolYOAK, K. J. (1989). Interdomain transfer between isomorphic topics in algebra and physics. Journal of Experimental Psychology: Learning, Memory, and Cognition, 15, pp. 153-166. http://dx.doi.org/10.1037/h0090398 http://dx.doi.org/10.1037/0278-7393.15.1.153

Campanario, J. M. y Moya, A. (1999). ¿Cómo enseñar Ciencias? Principales tendencias y propuestas. Enseñanza de las Ciencias, 17(2), pp. 179-192.

Concari, S. B. y Giorgi S. M. (2000). Los problemas resueltos en textos universitarios de Física. Enseñanza de las Ciencias, 18, pp. 381- 390

Furió, C. y Guisasola, J. (1997). Deficiencias epistemológicas en la enseñanza habitual de los conceptos de campo y potencial eléctrico. Enseñanza de las Ciencias, 15(2), pp. 259-271.

Furió, C. y J. Guisasola (1998). Dificultades de aprendizaje de los conceptos de carga y de campo eléctrico en estudiantes de bachillerato y universidad. Enseñanza de las Ciencias, 16(1), pp. 131-146.

Guisasola, J.; Salinas, J.; Almudi, J. M. y Velazco, S. (2003). Análisis de procesos de aplicación de las leyes de Gauss y Ampère por estudiantes universitarios de España y Argentina. Revista Brasileira de Ensino de Fisica, 25(2), pp. 195-206.

http://dx.doi.org/10.1590/S0102-47442003000200008

Guisasola, J.; Almudi, J. M. yZuZA, K. (2011). University Students' Understanding of Electromagnetic Induction. International Journal of Science Education,

http://dx.doi.org/10.1080/09500693.2011.624134

Halliday, D. y Resnick, R. (1982). Física. Parte 2. Mx: Compañía Editorial Continental, S. A.

Hsu, H. P. (1987). Análisis Vectorial. Addison-Wesley Iberoamericana.

Kelly, G. J. (2007). Discourse in Science Classrooms. En Abell y Lederman (eds.). Handbook of Research on Science Education, pp. 443-470. Londres: LEA, Publishers.

KIP, A. F. (1975). Fundamentos de Electricidad y Magnetismo. Mx: McGraw-Hill.

Krapas, S.; Alves, F. y Carvalho, L. R. (2000). Modelos Mentais e a Lei de Gauss. Investigaçóes em Ensino de Ciências, 5(1), pp. 7-21.

Leithold, L. (1998). El Cálculo. Oxford: Oxford University.

Manogue, C. A.; Browne, K.; Dray, T. y Edwards, B. (2006). Why is Ampère's law so hard? A look at middle-division physics. American Journal of Physics, 74(4), pp. 344-350. http://dx.doi.org/10.1119/1.2181179

Meredith, D. C. y Marrongelle, K. A. (2008). How students use mathematical resources in electrostatics context. American Journal of Physics, 76(6), pp. 570-578. http://dx.doi.org/10.1119/1.2839558

Merriam, S. B. (1998). Qualitative research and case study applications in education. San Francisco: Jossey - Bass Inc.

Pandiella, S.; Torné, P. C. y Macías, A. (2004). Las características de los textos de física y su incidencia en la comprensión. Investigaçoes em Ensino de Ciencias, 9(1).

Patton, M. Q. (1990). Qualitative Evaluation Methods. (2.a ed.). Thousand Oaks, Calif: Sage.

Piskunov, N. (1998). Cálculo diferencial e integral. México: Editorial Limusa.

Pocoví, M. C. (2007). The effects of a history-based instructional material on the students' understanding of field lines. Journal of Research in Science Teaching, 4(1), pp. 107-132. 
Pocoví, M. C. y Finley, F. (2003). Historical Evolution of the Field View and Textbook accounts. Science \& Education, 12(4), pp. 387-396.

Purcell, E. M. (1973). Electricidad y Magnetismo. España: McGraw-Hill Book Company.

Purcell, E. J.; Varberg, D. y Rigdon, S. E. (2001). Cálculo (8.a ed.). Mx: Pearson Educación.

Rabuffetti, H. T. (1984). Introducción al Análisis Matemático (Cálculo 2). Buenos Aires: El Ateneo.

Resnick, R.; Halliday, D. y Krane, K. (2009). Física. Vol. 2. México: Grupo Editorial Patria.

Rey Pastor, J.; Pi Calleja P. y Trejo, C. A. (1968). Análisis Matemático. Volumen II: Cálculo Infinitesimal de varias variables. Aplicaciones. (7. ${ }^{\mathrm{a}}$ ed.). Buenos Aires: Editorial Kapeluz.

SaArelainen, M. y Hirvonen, P. E. (2009). Designing a teaching sequence for electrostatics at undergraduate level by using educational reconstruction. Latin American Journal of Physics Education, 3(3), pp. 518-526.

Sadoski, M. y Paivio, A. (2004). A Dual Coding View of imagery and verbal processes in reading comprehension. En Ruddell, Ruddell y Singer (eds.). Theoretical Models and Processes of Reading (4. ${ }^{\mathrm{a}}$ ed.), pp. 1329-1362. Newark, DE: International Reading Association.

Schneider, M. B. (2004). Discovery-based Gauss Law. American Journal of Physics, 72(10), p. 1272. http://dx.doi.org/10.1119/1.1773174

Serway, R. y Jewett, J. Jr. (2009). Fisica para Ciencias e Ingeniería con Física Moderna. Vol. 2. México: Cengage Learning.

Sherin, B. L. (2001). How students understand Physics Equations. Cognition and Instruction, 19(4), pp. 479-541.

http://dx.doi.org/10.1207/S1532690XCI1904_3

Spiegel, M. (1970). Análisis Vectorial. Mx: Mc Graw Hill.

Spinadel, V. W. (2004). Cálculo 2. Buenos Aires: Nueva Librería.

Stewart, J. (2006). Cálculo Conceptos y contextos (3. a ed.). México: International Thomson Editores, S.A.

Tipler, P. y Mosca, G. (2010). Física para la Ciencia y la Tecnología, Electricidad y Magnetismo, luz. Vol. 2. México: Ed. Reverté.

Young, H. y Freedman, R. A. (2009). Física Universitaria con Física Moderna. Vol. 2. Mx: AddisonWesley.

Viennot, L. y Rainson, S. (1992). Students' reasoning about the superposition of electric fields. International Journal of Science Education, 14(4), pp. 475-487.

http://dx.doi.org/10.1080/0950069920140409

Viennot, L. y Rainson, S. (1999). Design and evaluation of research-based teaching sequence: the superposition of electric field. International Journal of Science Education, 21(1), pp. 1-16. http://dx.doi.org/10.1080/095006999290804

Wangsness, R. K. (1986). Electromagnetic Fields. Canadá: John Wiley \& Sons. 


\title{
Translation between symbolic languages in different content areas: the case of the electric field flux
}

\author{
M. Cecilia Pocoví, C. Collivadino \\ Universidad Nacional de Salta. Facultad de Ingeniería.Agencia de Promoción Científica y Tecnológica \\ Avda. Bolivia, 5150 (4400), Salta \\ cpocovi@unsa.edu.ar,collivad@unsa.edu.ar
}

A generic case study as that defined by (Merriam, 1998) is presented here. In the first part of the work, the symbolic system used in basic electromagnetism books when presenting the concept of electric flux is compared with the symbolic system used in calculus books when presenting Gauss' Theorem.

Learning from texts is one of the most important activities that students perform at the university level. In this work, we study the comprehension problems that can emerge during the first contact that students have with a text that is referred to the flux concept as it is treated in a Physics class. The concept is presented in the mathematical form of an equation. The learning of the concept of flux in the context of a university electromagnetism class has the peculiarity that it has already been learned by the students within the Calculus previous class.

In this work we have analyzed some comprehension problems that might have their roots in a lack of uniformity between the symbols used in Calculus and Physics classes. The analysis was guided by the Dual Coding Theory posed by Sadoski \& Paivio (2004). This model assumes that there are three dimensions or levels of information processing. The first one is called representational processing and allows the learning subject to recognize something as familiar. In the second level, the subject associates the new experience with a previous one. Even though these two first levels do not ensure a deep understanding of a concept, they constitute previous and necessary steps to allow it.

As the posed problem involves not only student's first understanding but also the symbology used in Calculus and Physics, this work has two sections.

In the first part of the work, the symbolic system used in basic electromagnetism books when presenting the concept of electric flux is compared with the symbolic system used in Calculus books when presenting Gauss' Theorem. The results show that, while there exists a great deal of uniformity within one area (Physics or Calculus), there exist discrepancies between areas.

The second part of this work deals with the symbolic-symbolic translation that students are able to carry out when having learned Gauss Theorem with a symbolic system that is different from the one used in Physics. The results show that many students have trouble recognizing some important symbols within the definition of flux. For example, many students interpret the symbol as a line integral. We postulate that this confusion is not due to the inability to recognize a symbol but, instead, constitutes a mis-recognition due to the fact that the same symbol has been used in a different way in previous Calculus classes.

It is suggested that the lack of interpretation of what the symbols mean can initially contribute to hinder comprehension about the flux concept.

Future investigations in this line should point to the design of instructional material that would help students in the recognition of symbols and operatory in their first encounter with the flux concept. In this sense, we are working on the development of a text that shows the parallelism between the two symbolic codes (that used in Calculus and the one used in Physics) for the first cases that students study with Gauss Law (point charge and spherical charge). 